\title{
Centralized and decentralized detection with cost-constrained measurements ${ }^{\text {is }}$
}

\author{
Eray Laz ${ }^{a, b}$, Sinan Gezici ${ }^{\text {a,* }}$ \\ ${ }^{a}$ Department of Electrical and Electronics Engineering, Bilkent University, Bilkent, Ankara 06800, Turkey \\ ${ }^{\mathrm{b}}$ Radar, Electronic Warfare and Intelligence Division, ASELSAN Inc., Ankara, Turkey
}

\section{A R T I C L E I N F O}

\section{Article history:}

Received 2 May 2016

Received in revised form

27 July 2016

Accepted 16 September 2016

Available online 19 September 2016

Keywords:

Hypothesis testing

Measurement cost

Decentralized detection

Centralized detection

Sensor networks

\begin{abstract}
A B S T R A C T
Optimal detection performance of centralized and decentralized detection systems is investigated in the presence of cost constrained measurements. For the evaluation of detection performance, Bayesian, Neyman-Pearson and J-divergence criteria are considered. The main goal for the Bayesian criterion is to minimize the probability of error (more generally, the Bayes risk) under a constraint on the total cost of the measurement devices. In the Neyman-Pearson framework, the probability of detection is to be maximized under a given cost constraint. In the distance based criterion, the J-divergence between the distributions of the decision statistics under different hypotheses is maximized subject to a total cost constraint. The probability of error expressions are obtained for both centralized and decentralized detection systems, and the optimization problems are proposed for the Bayesian criterion. The probability of detection and probability of false alarm expressions are obtained for the Neyman-Pearson strategy and the optimization problems are presented. In addition, $J$-divergences for both centralized and decentralized detection systems are calculated and the corresponding optimization problems are formulated. The solutions of these problems indicate how to allocate the cost budget among the measurement devices in order to achieve the optimum performance. Numerical examples are presented to discuss the results.
\end{abstract}

(c) 2016 Elsevier B.V. All rights reserved.

\section{Introduction}

In this paper, centralized and decentralized hypothesis-testing (detection) problems are investigated in the presence of cost constrained measurements. In such systems, decisions are performed based on measurements gathered by multiple sensors, the qualities of which are determined according to assigned cost values. The aim is to develop optimal cost allocation strategies for the Bayesian, Neyman-Pearson, and J-divergence criteria under a total cost constraint. In the case of centralized detection, a set of geographically separated sensors sends all of their measurements to a fusion center, and the fusion center decides on one of the hypotheses [1]. On the other hand, in decentralized detection, sensors transmit a summary of their measurements to the fusion center [2]. For quantifying the costs of measurement devices (sensors), the model in [3] is employed in this study. According to

${ }^{\mathrm{n}} \mathrm{Part}$ of this work was presented at the 17th IEEE International Workshop on Signal Processing Advances in Wireless Communications (SPAWC), Edinburgh, UK, July 2016.

* Corresponding author

E-mail addresses: eray@ee.bilkent.edu.tr (E. Laz), gezici@ee.bilkent.edu.tr (S. Gezici).
[3], the cost of a measurement device is basically determined by the number of amplitude levels that it can reliably distinguish. This cost model can be used in sensor network applications in which measurements are performed via various sensors. As an example, for fire detection in a forest, there can exist a finite number of sensors performing temperature measurements, and according to these measurements, the decision on the presence of fire is made. The accuracy of the decision depends on the quality of the measurements collected by the sensors. If the cost allocated to a sensor is higher, the measurement becomes less noisy as modeled in [3]. Similar applications can be considered in wireless cognitive radio, sonar and radar systems.

Detection and estimation problems considering system resource constraints have extensively been studied in the literature [4-22]. In [4], measurement cost minimization is performed under various estimation accuracy constraints. In [5], optimal distributed detection strategies are studied for wireless sensor networks by considering network resource constraints, where it is assumed that observations at the sensors are spatially and temporally independent and identically distributed (i.i.d.). Two types of constraints are taken into consideration related to the transmission power and the communication channel. For the communication channel, there exist two options, which are multiple access and 
parallel access channels. It is shown that using a multiple access channel with analog communication of local likelihood ratios (soft decisions) is asymptotically optimal when each sensor communicates with a constant power [5]. In [6], binary decentralized detection problem is investigated under the constraint of wireless channel capacity. It is proved that having a set of identical sensor is asymptotically optimal when the observations conditioned on the hypothesis are i.i.d. and the number of observations per sensor goes to infinity. In [7], a decentralized detection problem is studied, where the sensors have side information that affects the statistics of their measurements and the network has a cost constraint. The author examines wireless sensor networks with a cost constraint and a capacity constraint separately. In both scenarios, the error exponent is minimized under the specified constraints. The study in [7] produces a similar result to that in [6] for the scenario with the capacity constraint. In addition, $[7,8]$ have the same results for scenario with the power constraint. It is obtained that having identical sensors which use the same transmission scheme is asymptotically optimal when the observations are conditionally independent given the state of the nature.

In [9], the decentralized detection problem is studied in the presence of system level costs. These costs stem from processing the received signal and transmitting the local outputs to the fusion center. It is shown that the optimum detection performance can be obtained by performing randomization over the measurements and over the choice of the transmission time. In [10], the aim is to minimize the probability of error under communication rate constraints, where the sensors can censor their observations. The optimum result is obtained by censoring uninformative observations and sending informative observations to the fusion center. In [11], the aim is to obtain a network configuration that satisfies the optimum detection performance under a given cost constraint. The cost constraint depends on the number of sensors employed in the network. In [12], the optimal power allocation for distributed detection is studied, where both individual and joint constraints on the power that sensors use while transmitting their decisions to the fusion center are taken into consideration. The optimal detection performance is obtained for the proposed power allocation scheme. In [13], a binary hypothesis testing problem is investigated under communication constraints. The proposed algorithm determines a data reduction rate for transmitting a reduced version of data and finds the performance of the best test based on the reduced data. In [14], the decentralized detection problem is investigated under both power and bandwidth constraints. It is shown that combining many 'not so good' local decisions is better than combining a few very good local decisions in the case of large sensor systems. In [15-17], the decentralized detection problem is studied with fusion of Gaussian signals. It is stated that there is an optimal number of local sensors that achieves the highest performance under a given global power constraint, and increasing the number of sensors beyond the optimal number degrades the performance. In [18], the authors investigate decentralized detection and fusion performance of a sensor network under a total power constraint. It is shown that using non-orthogonal communication between local sensors and the fusion center improves fusion performance monotonically. In [19], the optimization of detection performance of a sensor network is studied under communication constraints, and it is found that the optimal fusion rule is similar to the majority-voting rule for binary decentralized detection. In [21], the sensor (or, sample) selection problem is studied for distributed detection. The authors seek the best subset of data samples that results in a desired detection probability. To this aim, the number of selected sensors that perform the sensing task is minimized under a given probability of error constraint for the Bayesian criterion and under false-alarm and miss-detection rate constraints for the Neyman-Pearson criterion. In addition, a dual problem is also proposed such that the probability of error is minimized for a constant number of selected sensors in the Bayesian criterion. For the Neyman-Pearson criterion, it is aimed to minimize the probability of miss detection under a given false alarm constraint and a fixed number of selected sensors. It is found that for conditionally independent observations, the best sensors are the ones with the largest local average log-likelihood ratio and the smallest local average root-likelihood ratio in the NeymanPearson and Bayesian setting, respectively. As in [21], the sensor selection problem is studied in [22], where the aim is to find a subset of $p$ out of $n$ sensors that yield the best detection performance. The authors show numerically the validity of the Chernoff and Kullback-Leibler sensor selection criteria by illustrating that they lead to sensor selection strategies that are nearly optimal both in the Bayesan and Neyman-Pearson sense.

Based on the cost function proposed in [3] for obtaining measurements, various studies have been performed on estimation with cost constraints [4,20]. In particular, Ref. [4] considers the costs of measurements and aims to minimize the total cost under various estimation accuracy constraints. In [20], average Fisher information maximization is studied under cost constrained measurements. On the other hand, Ref. [23] investigates the tradeoff between reducing the measurement cost and keeping the estimation accuracy within acceptable levels in continuous time linear filtering problems. In [24], the channel switching problem is studied, where the aim is to minimize the probability of error between a transmitter and a receiver that are connected via multiple channels and only one channel can be used at a given time. In that study, a logarithmic cost function similar to that in [3] is employed for specifying the cost of using a certain channel.

Although costs of measurements have been considered in various estimation and channel switching problems such as $[4,20,23,24]$, there exist no studies in the literature that consider the optimization of both centralized and decentralized detection systems in the presence of cost constrained measurements based on a specific cost function as in [3]. In this study, we first consider the centralized detection problem and propose a general formulation for allocating the cost budget to measurement devices in order to achieve the optimum performance according to the Bayesian criterion. Also, a closed-form expression is obtained for binary hypothesis testing with Gaussian observations and generic prior probabilities. In addition, it is shown that the probability of error expression for the Gaussian case is convex with respect to the total cost constraint in the case of equally likely binary hypotheses (Lemma 1). Then, we investigate the decentralized detection problem in the Bayesian framework with some common fusion rules, and present a generic formulation that aims to minimize the probability of error by optimally allocating the cost budget to measurement devices. A numerical solution is proposed for binary hypothesis testing with Gaussian observations. As convexity is an important property for the optimization problems, the convexity property is explored for the case of two measurement devices (Lemma 2). Furthermore, the Neyman-Pearson and $J$-divergence criteria are investigated for the cost allocation problem in order to achieve the optimum detection performance. The general optimization problems are proposed for both criteria and the Gaussian scenario is investigated as a special case. As for the Bayesian criterion, both centralized and decentralized detection systems are taken into consideration.

The remainder of the paper is organized as follows: In Section 2, the optimal cost allocation among measurement devices is studied for the Bayesian criterion. In Section 3, the problem is investigated in the Neyman-Pearson framework. In Section 4, the optimization problems obtained according to $J$-divergence are examined. In Section 5, numerical examples that illustrate the obtained results are presented. Finally, conclusions are presented in Section 6. 


\section{Cost allocation for Bayesian criterion}

In this section, the cost allocation problem is investigated for hypothesis-testing problems based on the Bayesian criterion. When it is possible to assign costs to the decisions and when the prior probabilities of the states of nature are known, the Bayesian approach is a well-suited candidate for detection criterion [25]. The aim in this section is to minimize the Bayes risk for both centralized and decentralized detection systems under a total cost constraint on measurements.

\subsection{Centralized detection}

In centralized detection problems, all sensor nodes transmit their observations to the fusion center, and the decision is performed in the fusion center based on the data from all the sensors. The system model for centralized detection is shown in Fig. 1.

As illustrated in Fig. $1, x_{1}, x_{2}, \ldots, x_{K}$ represent the scalar observations, and $s_{1}, s_{2}, \ldots, s_{K}$ denote the sensors by which the measurements are taken. The measurement at sensor $i$ is represented as $y_{i}=x_{i}+m_{i}$, where $m_{i}$ is the measurement noise. The measurement $\mathbf{y} \in \mathbb{R}^{K}$ is processed by the fusion center to produce the final decision $\gamma(\mathbf{y})$, where $\mathbf{y}=\left[y_{1}, y_{2}, \ldots, y_{K}\right]^{T}$ and $\gamma(\mathbf{y})$ takes values from $\{0,1, \ldots, M-1\}$ for $M$-ary hypothesis testing.

In the Bayesian hypothesis-testing framework, the optimum decision rule is the one that minimizes the Bayes risk, which is defined as the average of the conditional risks [25]. The conditional risk for a decision rule $\delta(\cdot)$ when the state of nature is $H_{j}$ is given by

$R_{j}(\delta)=\sum_{i=0}^{M-1} \tilde{c}_{i j} P_{j}\left(\Gamma_{i}\right)$

where $\tilde{c}_{i j}$ is the cost of choosing hypothesis $H_{i}$ when the state of nature is $H_{j}$, and $P_{j}\left(\Gamma_{i}\right)$ is the probability of deciding hypothesis $H_{i}$ when $H_{j}$ is correct, with $\Gamma_{\mathrm{i}}$ denoting the decision region for hypothesis $H_{i}$. Then, the Bayes risk can be expressed as

$r(\delta)=\sum_{j=0}^{M-1} \pi_{j} R_{j}(\delta)$

where $\pi_{\mathrm{j}}$ is the prior probability of hypothesis $H_{j}$. For the values of $\tilde{c}_{i j}$, uniform cost assignment (UCA) is commonly employed, which is stated as [25]

$\tilde{c}_{i j}= \begin{cases}0, & \text { if } i=j, \\ 1, & \text { if } i \neq j .\end{cases}$

For UCA, the Bayes rule, which minimizes the Bayes risk specified by (1) and (2), reduces to choosing the hypothesis with the maximum a posteriori probability (MAP), and the corresponding Bayes risk can be stated, after some manipulation, as

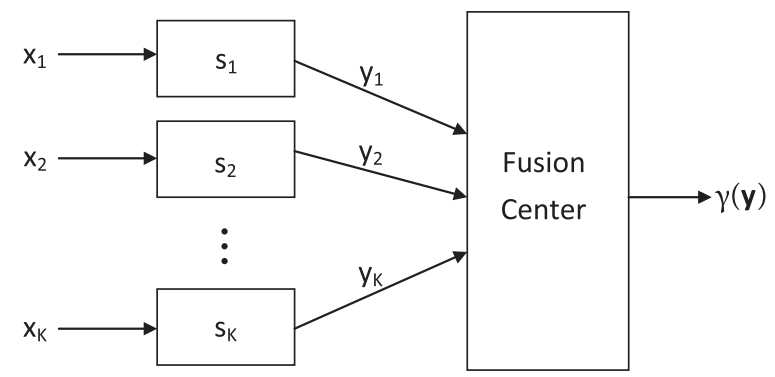

Fig. 1. Centralized detection system model.
$r\left(\delta_{B}\right)=1-\int_{\mathbb{R}^{K}} \max _{l=\{0,1, \ldots, M-1\}} \pi_{l} p_{l}(\mathbf{y}) d \mathbf{y}$,

where $\delta_{B}$ denotes the Bayes rule, and $p_{l}(\mathbf{y})$ is the probability distribution of $\mathbf{y}$ under hypothesis $H_{l}$ [25].

In this section, the aim is to perform the optimal cost allocation among the sensors in Fig. 1 in order to minimize the Bayes risk expression in (4) under a total cost constraint. The cost of measuring the $i$ th component of the observation vector, $x_{i}$, is given by $C_{i}=0.5 \log _{2}\left(1+\sigma_{x_{i}}^{2} / \sigma_{m_{i}}^{2}\right)$, where $\sigma_{x_{i}}^{2}$ is the variance of $x_{i}$ and $\sigma_{m_{i}}^{2}$ is the variance of the noise introduced by the $i$ th sensor [3]. Then, the total cost is expressed as

$C=\sum_{i=1}^{K} C_{i}=\frac{1}{2} \sum_{i=1}^{K} \log _{2}\left(1+\frac{\sigma_{x_{i}}^{2}}{\sigma_{m_{i}}^{2}}\right)$.

As mentioned in Section 1, the number of amplitude levels that can be distinguished by the measurement device determines the cost of the measurement. The dynamic range of the input to the measurement devices has no effect on the cost of the measurements provided that the number of resolvable levels stays the same. The cost function in (5) uses the variances of the observation and the measurement noise to describe the number of distinguishable amplitude levels [3]. This is the same motivation as that used by Hartley [26]. Moreover, the cost function has the same form as Shannon's capacity formula for the Gaussian noise channel [27], where $x_{i}$ is transmitted across a communication channel that adds a noise term $m_{i}$ to it. Apart from these, the cost function for each sensor is monotonically decreasing, nonnegative, and convex with respect to $\sigma_{m_{i}}^{2}$ for $\forall \sigma_{m_{i}}^{2}>0$ and $\forall \sigma_{x_{i}}^{2}>0$. (The convexity property of the cost function can easily be shown by examining its Hessian matrix [28].) In addition, when the measurement noise variance is low, the cost is high since the number of amplitude levels that the device can distinguish gets high [3]. When $\sigma_{m_{i}}^{2}$ goes to infinity, the cost converges to zero and when $\sigma_{m_{i}}^{2}$ goes to zero, the cost approaches infinity.

Based on (4) and (5), the following optimization problem is proposed for centralized detection problems:

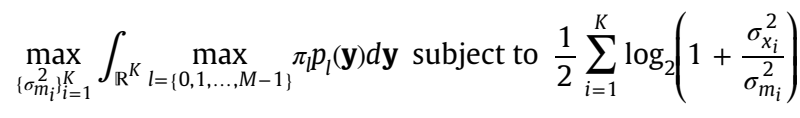

$\leq C_{T}$,

where $C_{T}$ is the (total) cost constraint. Hence, the optimal allocation of the measurement noise variances, $\sigma_{m_{i}}^{2}$, (equivalently, the costs, $C_{i}$ ) is to be performed under the total cost constraint. It is also noted that the maximization of the objective function in (6) corresponds to the minimization of the Bayes risk in (4), which represents the probability of error for the Bayes rule. When the optimization problem proposed in (6) is solved, the optimum cost values for the measurement devices (sensors) are obtained and these values achieve the optimum performance for centralized detection.

In practical systems, the observations, $\mathbf{x}=\left[x_{1}, \ldots, x_{K}\right]^{T}$, are independent of the measurement noise, $\mathbf{m}=\left[m_{1}, \ldots, m_{K}\right]^{T}$. Hence, the conditional probability density function (PDF) of the measurement vector when hypothesis $H_{l}$ is true can be obtained as the convolution of the PDFs of $\mathbf{m}$ and $\mathbf{x}$ as follows:

$p_{l}(\mathbf{y})=\int_{\mathbb{R}^{K}} p_{\mathbf{M}}(\mathbf{m}) p_{\mathbf{X}}\left(\mathbf{y}-\mathbf{m} \mid H_{l}\right) d \mathbf{m}$.

In addition, if the sensors have independent noise, $p_{\mathbf{M}}(\mathbf{m})$ can be expressed as $p_{\mathbf{M}}(\mathbf{m})=p_{M_{1}}\left(m_{1}\right) \cdots p_{M_{K}}\left(m_{K}\right)$.

As a special case, a centralized binary hypothesis-testing problem is investigated in the presence of Gaussian observations and 
measurement noise, which is a common scenario in practice. In this case, the distribution of observation $\mathbf{x}$ under hypothesis $H_{0}$ is Gaussian with mean vector $\boldsymbol{\mu}_{\mathbf{0}}$ and covariance matrix $\boldsymbol{\Sigma}$, which is denoted by $\mathcal{N}\left(\mu_{0}, \boldsymbol{\Sigma}\right)$. Similarly, $\mathbf{x}$ is distributed as $\mathcal{N}\left(\boldsymbol{\mu}_{\mathbf{1}}, \boldsymbol{\Sigma}\right)$ under hypothesis $H_{1}$. In addition, the measurement noise vector, $\mathbf{m}$, is distributed as $\mathcal{N}\left(\mathbf{0}, \boldsymbol{\Sigma}_{\mathbf{m}}\right)$, where $\boldsymbol{\Sigma}_{\mathbf{m}}=\operatorname{diag}\left\{\sigma_{m_{1}}^{2}, \sigma_{m_{2}}^{2}, \ldots, \sigma_{m_{K}}^{2}\right\}$; that is, the measurement noise is independent for different sensors [3]. Considering that $\mathbf{x}$ and $\mathbf{m}$ are independent, the distribution of the measurement, $\mathbf{y}=\mathbf{x}+\mathbf{m}$, is denoted by $\mathcal{N}\left(\boldsymbol{\mu}_{\mathbf{0}}, \boldsymbol{\Sigma}+\boldsymbol{\Sigma}_{\mathbf{m}}\right)$ under hypothesis $H_{0}$ and by $\mathcal{N}\left(\boldsymbol{\mu}_{\mathbf{1}}, \boldsymbol{\Sigma}+\boldsymbol{\Sigma}_{\mathbf{m}}\right)$ under $H_{1}$.

For the hypothesis-testing problem specified in the previous paragraph, the Bayes risk corresponding to the Bayes rule can be obtained as follows in the case of UCA [25, Chapter 3]:

$r\left(\delta_{B}\right)=\pi_{0} Q\left(\frac{\ln \left(\pi_{0} / \pi_{1}\right)}{d}+\frac{d}{2}\right)+\pi_{1} Q\left(\frac{d}{2}-\frac{\ln \left(\pi_{0} / \pi_{1}\right)}{d}\right)$,

where

$d \triangleq \sqrt{\left(\mu_{1}-\mu_{0}\right)^{T}\left(\Sigma+\Sigma_{\mathbf{m}}\right)^{-1}\left(\mu_{1}-\mu_{0}\right)}$

and $Q(x)=(1 / \sqrt{2 \pi}) \int_{x}^{\infty} \mathrm{e}^{-0.5 t^{2}} d t$ denotes the $Q$-function.

It can be shown that the derivative of $r\left(\delta_{B}\right)$ in (8) with respect to $d$ is negative for all values of $d$; hence, $r\left(\delta_{B}\right)$ is a monotone decreasing function of $d$. Therefore, the minimization of $r\left(\delta_{B}\right)$ can be achieved by maximizing $d$. If the observations are assumed to be independent; that is, if $\boldsymbol{\Sigma}=\operatorname{diag}\left\{\sigma_{x_{1}}^{2}, \sigma_{x_{2}}^{2}, \ldots, \sigma_{x_{K}}^{2}\right\}$, then $d$ can be expressed as

$d=\sqrt{\sum_{i=1}^{K} \frac{\mu_{i}^{2}}{\sigma_{x_{i}}^{2}+\sigma_{m_{i}}^{2}}}$,

where $\mu_{\mathrm{i}}$ represents the $i$ th component of the vector $\boldsymbol{\mu}_{\mathbf{1}}-\boldsymbol{\mu}_{\mathbf{0}}$.

Hence, the optimization problem in (6) for this case is stated as follows:

$\max _{\left\{\sigma_{m_{i}}^{2}\right\}_{i=1}^{K}} \sum_{i=1}^{K} \frac{\mu_{i}^{2}}{\sigma_{x_{i}}^{2}+\sigma_{m_{i}}^{2}}$ subject to $\frac{1}{2} \sum_{i=1}^{K} \log _{2}\left(1+\frac{\sigma_{x_{i}}^{2}}{\sigma_{m_{i}}^{2}}\right) \leq C_{T}$.

The objective function in (11) is convex with respect to $\sigma_{m_{i}}^{2}$ for $\forall \sigma_{m_{i}}^{2}>0$ and $\forall \sigma_{x_{i}}^{2}>0$ since the Hessian matrix of the objective function,

$\mathbf{H}=\operatorname{diag}\left\{2 \mu_{1}^{2} /\left(\sigma_{x_{1}}^{2}+\sigma_{m_{1}}^{2}\right)^{3}, 2 \mu_{2}^{2} /\left(\sigma_{x_{2}}^{2}+\sigma_{m_{2}}^{2}\right)^{3}, \ldots, 2 \mu_{K}^{2} /\left(\sigma_{x_{K}}^{2}+\sigma_{m_{K}}^{2}\right)^{3}\right\}, \quad$ is positive definite. Since a convex objective function is maximized over a convex set, the solution lies at the boundary $[20,29]$. Therefore, the constraint function becomes an equality constraint and the optimization problem can be solved by using the Lagrange multipliers method $[28,29]$. Based on this approach, the optimal cost allocation algorithm is obtained as follows:

$\sigma_{m_{i}}^{2}= \begin{cases}\frac{\sigma_{x_{i}}^{4}}{\mu_{i}^{2} \alpha-\sigma_{x_{i}}^{2}}, & \text { if } \sigma_{x_{i}}^{2}<\mu_{i}^{2} \alpha \\ \infty, & \text { if } \sigma_{x_{i}}^{2} \geq \mu_{i}^{2} \alpha\end{cases}$

with

$\alpha=\left(2^{2 C_{T}} \prod_{i \in S_{K}} \frac{\sigma_{x_{i}}^{2}}{\mu_{i}^{2}}\right)^{1 /\left|S_{K}\right|}$,

where set $S_{K}$ is given by $S_{K}=\left\{i \in\{1,2, \ldots, K\}: \sigma_{m_{i}}^{2} \neq \infty\right\}$, and $\left|S_{K}\right|$ represents the number of elements in the set $S_{K}$. The algorithm in (12) implies that if the observation variance $\sigma_{x_{i}}^{2}$ is greater than $\mu_{i}^{2} \alpha$, the variance of the measurement device (sensor) is set to infinity; that is, the observation is not measured at all, and the cost of the measurement device is zero. If the observation variance is smaller than the specified threshold, the variance of the measurement noise is calculated according to the expression in (12), which states that if the observation variance is low, the variance of the measurement device is assigned to be low. In other words, if the observation variance is low, a device with a high cost is considered to take measurements. Moreover, if the difference between the means of the observations for the two hypotheses, $\mu_{\mathrm{i}}$, is high and $\sigma_{x_{i}}^{2}<\mu_{i}^{2} \alpha$ is satisfied, a low measurement noise variance is assigned to the measurement device. If $\mu_{\mathrm{i}}$ is close to zero such that $\sigma_{x_{i}}^{2} \geq \mu_{i}^{2} \alpha$, a measurement device with zero cost is considered. Apart from this, if the observations are i.i.d. given the hypothesis, the variances of the measurement devices are chosen as equal, meaning that all the devices are required to have equal costs in order to achieve the optimum performance. The variances of the measurement devices become $\sigma_{m}^{2}=\sigma_{x}^{2} /\left(2^{2 C_{T} / K}-1\right)$ for i.i.d. observations.

In the following lemma, the probability of error corresponding to the optimal cost allocation in (12) is shown to be convex with respective to the total cost constraint, $C_{T}$, for the case of equal priors.

Lemma 1. Consider a binary hypothesis-testing problem in the presence of independent Gaussian observations and measurement noise. Then, for the optimal cost allocation strategy in (12), the probability of error in (8) is a convex monotone decreasing function of the total cost constraint $C_{T}$ in the case of equal priors; i.e., $\pi_{0}=\pi_{1}=0.5$.

Proof. In the case of equal priors, the probability of error in (8) reduces to $Q(d / 2)$. Assume, without loss of generality, that the first $N$ of $K$ sensors have finite measurement noise variances; that is, $\sigma_{m_{i}}^{2}<\infty$ for $i \in\{1, \ldots, N\}$. Then, from (10), the probability of error can be written as $P_{e}=Q\left(\frac{1}{2} \sqrt{\sum_{i=1}^{N} \frac{\mu_{i}^{2}}{\sigma_{x_{i}}^{2}+\sigma_{m_{i}}^{2}}}\right)$. When the optimal $\sigma_{m_{i}}^{2}$ values obtained from (12) and (13) are inserted into the probability of error expression, the optimal probability of error is stated as

$P_{e}^{*}=Q\left(\frac{1}{2} \sqrt{\left(\sum_{i=1}^{N} \frac{\mu_{i}^{2}}{\sigma_{x_{i}}^{2}}\right)-\tau 2^{-2 C_{T} / N}}\right)$,

where $\tau \triangleq N\left(\frac{\mu_{1}^{2} \cdots \mu_{N}^{2}}{\sigma_{x_{1}}^{2} \cdots \sigma_{x_{N}}^{2}}\right)^{1 / N}$. The first order derivative of $P_{e}^{*}$ with respect to the total cost $C_{T}$ is obtained as

$\frac{\partial P_{e}^{*}}{\partial C_{T}}=-\frac{(\ln 2) \tau 2^{-2 C_{T} / N} \exp \left(-\left(\beta-\tau 2^{-2 C_{T} / N}\right) / 8\right)}{2 \sqrt{2 \pi} N \sqrt{\beta-\tau 2^{-2 C_{T} / N}}}$,

where $\beta \triangleq \frac{\mu_{1}^{2}}{\sigma_{\chi_{1}}^{2}}+\cdots+\frac{\mu_{N}^{2}}{\sigma_{\chi_{N}}^{2}}$. Then, the second order derivative of $P_{e}^{*}$ with respect to the total $\operatorname{cost} C_{T}$ is calculated, after some manipulation, as follows:

$$
\begin{aligned}
\frac{\partial^{2} P_{e}^{*}}{\partial C_{T}^{2}}= & \frac{\tau}{\sqrt{2 \pi}}\left(\frac{\ln 2}{N}\right)^{2} 2^{-4 C_{T} / N}\left(\beta-\tau 2^{-2 C_{T} / N}\right)^{-1 / 2} \\
& \exp \left(-\frac{\left(\beta-\tau 2^{-2 C_{T} / N}\right)}{8}\right)\left(\frac{\tau}{8}+2^{2 C_{T} / N}+\frac{\tau}{2}\left(\beta-\tau 2^{-2 C_{T} / N}\right)^{-1}\right) .
\end{aligned}
$$

As the arithmetic mean is larger than or equal to the geometric mean, $\beta \geq \tau$ is obtained. Then, $\beta>\tau 2^{-2 C_{T} / N}$ since $2^{-2 C_{T} / N}<1$. Therefore, it is observed from (15) and (16) that the first and the second order derivatives of $P_{e}^{*}$ with respect to $C_{T}$ are negative and positive, respectively. Hence, $P_{e}^{*}$ is a convex and monotone decreasing function of the total cost constraint $C_{T}$ for all $C_{T}>0$.

Lemma 1 states the convexity property of the probability of 
error corresponding to the optimal cost allocation strategy in (12) for equally likely binary hypotheses and in the presence of independent Gaussian observations and measurement noise. It should be noted that the convexity property in Lemma 1 is specific for the case of equal priors and non-convex behavior can be observed for some $C_{T}$ for hypotheses with unequal priors.

At this step, it is important to express the dual of the problem, which aims to find the minimum total measurement cost under the required detection performance. The optimization problem for the case in Lemma 1 can be written as follows:

$\min _{\substack{\left.\sigma_{m_{i}}^{2}\right\}_{i=1}^{K} \\ 2}} \frac{1}{2} \sum_{i=1}^{K} \log _{2}\left(1+\frac{\sigma_{x_{i}}^{2}}{\sigma_{m_{i}}^{2}}\right)$ subject to $Q\left(\frac{1}{2} \sqrt{\sum_{i=1}^{K} \frac{\mu_{i}^{2}}{\sigma_{x_{i}}^{2}+\sigma_{m_{i}}^{2}}}\right) \leq P_{e c}$

where $P_{e c}$ represents the probability of error constraint. The Lagrange multipliers method is used in order to solve the problem in (17) as in the solution of the problem in (11). Then, the optimal cost allocation strategy achieving the minimum total measurement cost under the given probability of error constraint is obtained as follows:

$\sigma_{m_{i}}^{2}= \begin{cases}\frac{\sigma_{x_{i}}^{4}}{\mu_{i}^{2} \xi-\sigma_{x_{i}}^{2}}, & \text { if } \sigma_{x_{i}}^{2}<\mu_{i}^{2} \xi \\ \infty, & \text { if } \sigma_{x_{i}}^{2} \geq \mu_{i}{ }^{2} \xi\end{cases}$

with

$\xi=\frac{\left|S_{K}\right|}{\left(\sum_{i \in S_{K}} \frac{\mu_{i}^{2}}{\sigma_{x_{i}}^{2}}\right)-4\left(Q^{-1}\left(P_{e c}\right)\right)^{2}}$,

where $Q^{-1}(\cdot)$ represents the inverse of the $Q$-function.

\subsection{Decentralized detection}

In contrast to centralized detection, local sensors send a summary of their observations to the fusion center in decentralized detection. For binary hypothesis-testing, local sensors can send their binary decisions about the true hypothesis ( 0 or 1 ) to the fusion center. The fusion center collects the binary decisions of the sensors and decides on the hypothesis. The fusion center can employ, e.g., OR, AND, or majority rules [30], as discussed in the following. The system model in this scenario is presented in Fig. 2. As in centralized detection, sensor $i, s_{i}$, measures the observation as $y_{i}=x_{i}+m_{i}$. Then, the sensors make local decisions about one of the two hypotheses as $\gamma_{i}\left(y_{i}\right)=u_{i}$, where $u_{i}$ is equal to 0 for hypothesis $H_{0}$ and 1 for hypothesis $H_{1}$. The outputs of the sensors, $u_{1}, u_{2}, \ldots, u_{K}$, are provided as inputs to the fusion center, which makes the final decision denoted by $\Gamma(\mathbf{u})$. The fusion rule that is employed in this section is the majority rule [30]. The majority rule is optimal when the noise components of the sensors are i.i.d., the hypotheses are equally likely, and the observations are i.i.d. and independent of the noise of the sensors [31]. The expression

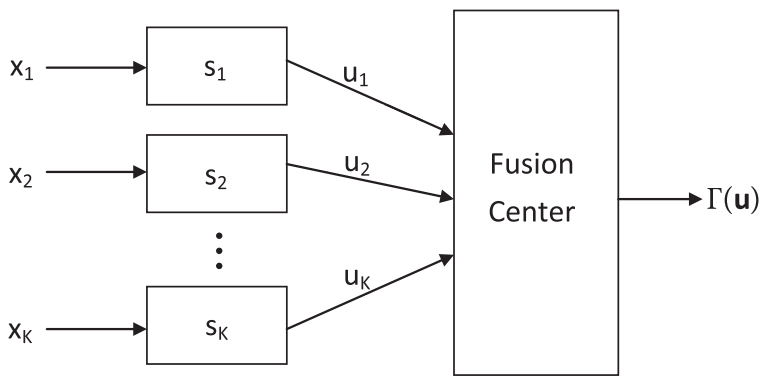

Fig. 2. Decentralized detection system model. for the majority rule is given by

$\Gamma\left(u_{1}, u_{2}, \ldots, u_{K}\right)= \begin{cases}1, & \text { if } \sum_{i=1}^{K} u_{i} \geq t \\ 0, & \text { if } \sum_{i=1}^{K} u_{i}<t\end{cases}$

with $t=\lfloor K / 2\rfloor+1$, where $\lfloor\cdot\rfloor$ represents the floor operator that maps a real number to the largest integer lower than or equal to itself. Although the majority rule is considered in the following analysis, the results can easily be extended for generic integer values of $t$ in (20). (For $t=1$ and $t=K$, the rule in (20) reduces to the OR fusion rule and the AND fusion rule, respectively.)

Considering independent but not necessarily identically distributed measurements $\left(y_{i}\right.$ 's), the probability of error (i.e., the Bayes risk for UCA) for the fusion rule in (20) can be calculated as

$r(\Gamma)=\pi_{0} \sum_{z=t}^{K} \sum_{c=1}^{\left(\begin{array}{c}K \\ z\end{array}\right)} \prod_{i=1}^{K} p_{l_{(z, c, i)}^{i}}^{i}+\pi_{1} \sum_{z=0}^{t-1} \sum_{c=1}^{\left(\begin{array}{c}K \\ z\end{array}\right)} \prod_{i=1}^{K} p_{l_{(z, c, i)}^{i}}^{i}$,

where $p_{l_{(z, c, i j} j}^{i}$ denotes, for the ith sensor, the probability of choosing hypothesis $H_{l_{(z, c, i)}}$ when hypothesis $H_{j}$ is true, and $l_{(z, c, i)}$ corresponds to the element at the $c$ th row and the ith column of matrix $\mathbf{L}(z)$, which has a dimension of $\left(\begin{array}{l}K \\ z\end{array}\right) \times K$ and is formed as follows: The numbers of 1 's and 0 's in a row are $z$ and $K-z$, respectively, and the rows of the matrix contain all possible combinations of $z 1$ 's and $K-z$ 0's. For example, matrix $\mathbf{L}(z)$ for $K=5$ and $z=3$ can be given as follows:

$\mathbf{L}(z)=\left(\begin{array}{lllll}1 & 1 & 1 & 0 & 0 \\ 1 & 1 & 0 & 0 & 1 \\ 0 & 1 & 1 & 1 & 0 \\ 0 & 0 & 1 & 1 & 1 \\ 1 & 0 & 1 & 0 & 1 \\ 1 & 1 & 0 & 1 & 0 \\ 0 & 1 & 0 & 1 & 1 \\ 0 & 1 & 1 & 0 & 1 \\ 1 & 0 & 1 & 1 & 0 \\ 1 & 0 & 0 & 1 & 1\end{array}\right)$,

where, e.g., $l_{(3,1,3)}=1, l_{(3,4,2)}=0$, and $l_{(3,3,3)}=1$. Although matrix $\mathbf{L}(z)$ is not unique (e.g., the orders of the rows can be changed), all the $\mathbf{L}(z)$ matrices result in the same probability of error in (21).

For the case of i.i.d. measurements $\left(y_{i}^{\prime} \mathrm{s}\right)$ and identical decision rules at the sensors, the probability of error for the fusion rule in (20) can be expressed, as a special case of (21), as follows:

$r(\Gamma)=\pi_{0} \sum_{z=t}^{K}\left(\begin{array}{l}K \\ z\end{array}\right)\left(p_{10}\right)^{z}\left(p_{00}\right)^{K-z}+\pi_{1} \sum_{z=0}^{t-1}\left(\begin{array}{l}K \\ z\end{array}\right)\left(p_{11}\right)^{z}\left(p_{01}\right)^{K-z}$,

where $p_{l j}$ represents, for each sensor, the probability of deciding for hypothesis $H_{l}$ when hypothesis $H_{j}$ is true.

In the decentralized detection framework, the aim is to minimize the probability of error in (21) under the total cost constraint; that is,

$$
\begin{aligned}
& \min _{\left\{\sigma_{m_{i}}^{2}\right\}_{i=1}^{K}} \pi_{0} \sum_{z=t}^{K} \sum_{c=1}^{\left(\begin{array}{c}
K \\
z
\end{array}\right)} \prod_{i=1}^{K} p_{l_{(z, c, i)}^{i} 0}^{i} \\
& +\pi_{1} \sum_{z=0}^{t-1} \sum_{c=1}^{K} \prod_{i=1}^{K} p_{l_{(z, c, i)}}^{i} \text { subject to } \frac{1}{2} \sum_{i=1}^{K} \log _{2}\left(1+\frac{\sigma_{x_{i}}^{2}}{\sigma_{m_{i}}^{2}}\right) \leq C_{T} .
\end{aligned}
$$

In order to solve this optimization problem, the conditional probability densities are obtained and inserted in the objective function. Then, an exhaustive search is applied to find the measurement noise variances. In order to reduce the computation 
time, parallel computing can be used. The solution of (23) provides the optimum cost allocation strategy for the considered decentralized detection system.

As a special case, the Gaussian scenario is investigated. Suppose that the probability distributions of the observations are independent when the hypothesis is given, and the distribution of the $i$ th observation is denoted by $\mathcal{N}\left(\mu_{i 0}, \sigma_{x_{i}}^{2}\right)$ and $\mathcal{N}\left(\mu_{i 1}, \sigma_{x_{i}}^{2}\right)$ under hypothesis $H_{0}$ and hypothesis $H_{1}$, respectively. In addition, the distribution of the $i$ th measurement noise is given by $\mathcal{N}\left(0, \sigma_{m_{i}}^{2}\right)$, and the observations are independent of the measurement noise. For the sensors, the Bayes rule is employed assuming UCA and equally likely priors [25]. In this setting, the probability distribution of $u_{i}$ (i.e., the decision of the $i$ th sensor) given the hypotheses can be specified as follows:

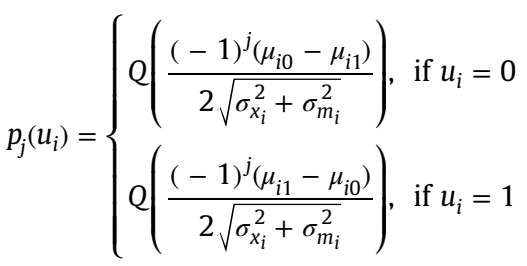

for $j \in\{0,1\}$, where $p_{j}\left(u_{i}\right)$ represents the probability of $u_{i}$ under hypotheses $H_{j}$. Hence, the optimization problem can be expressed for the Gaussian case as follows:

$$
\begin{aligned}
& \min _{\left\{\begin{array}{l}
\left.\sigma_{m_{i}}^{2}\right\}_{i=1}^{K} \\
2
\end{array}\right.} \frac{1}{2} \sum_{z=t}^{K} \sum_{c=1}^{\left(\begin{array}{l}
K \\
z
\end{array}\right)} \prod_{i=1}^{K} Q\left(\beta_{(z, c, i)} \frac{\mu_{i 1}-\mu_{i 0}}{2 \sqrt{\sigma_{x_{i}}^{2}+\sigma_{m_{i}}^{2}}}\right) \\
& +\frac{1}{2} \sum_{z=0}^{t-1} \sum_{c=1}^{\left(\begin{array}{l}
K \\
z
\end{array}\right)} \prod_{i=1}^{K} Q\left(-\beta_{(z, c, i)} \frac{\mu_{i 1}-\mu_{i 0}}{2 \sqrt{\sigma_{x_{i}}^{2}+\sigma_{m_{i}}^{2}}}\right) \text { subject to } \frac{1}{2} \\
& \sum_{i=1}^{K} \log _{2}\left(1+\frac{\sigma_{x_{i}}^{2}}{\sigma_{m_{i}}^{2}}\right) \leq C_{T},
\end{aligned}
$$

where $\beta_{(z, c, i)}=2 l_{(z, c, i)}-1$. The solution of this optimization problem leads to the optimal performance for the considered decentralized detection system by optimally allocating the cost values to the measurement devices (sensors).

Remark 1. The decisions at the local sensors are made according to the Bayesian criterion and the optimization is performed for the given fusion rule, which is the majority rule.

In the following lemma, the convexity of the optimization problem in (25) is investigated for the special case of two sensors.

Lemma 2. Consider the Gaussian scenario that leads to the optimization problem in (25). In addition, suppose that $K=2, \mu_{i 0}=0$, and $\mu_{i 1}=\mu>0$ for $i=1,2$. Then, the problem in (25) is a convex optimization problem if $\sigma_{x_{i}}^{2}+\sigma_{m_{i}}^{2} \leq \mu^{2} / 12$ for $i=1,2$ and for all values of $\sigma_{m_{i}}^{2}$ under the total cost constraint.

Proof. Under the assumptions specified in the lemma, the objective function in (25) can be expressed as

$$
\begin{aligned}
r(\Gamma)= & \frac{1}{2} Q\left(\frac{\mu}{2 \sqrt{\sigma_{x_{1}}^{2}+\sigma_{m_{1}}^{2}}}\right) Q\left(\frac{\mu}{2 \sqrt{\sigma_{x_{2}}^{2}+\sigma_{m_{2}}^{2}}}\right) \\
& +\frac{1}{2}\left(1-Q\left(-\frac{\mu}{2 \sqrt{\sigma_{x_{1}}^{2}+\sigma_{m_{1}}^{2}}}\right) Q\left(-\frac{\mu}{2 \sqrt{\sigma_{x_{2}}^{2}+\sigma_{m_{2}}^{2}}}\right)\right) .
\end{aligned}
$$

The Hessian matrix $\mathbf{H}$ of $r(\Gamma)$ is stated as follows:
$\mathbf{H}=\left(\begin{array}{cc}r_{\sigma_{m_{1}}^{2}, \sigma_{m_{1}}^{2}}^{2} & r_{\sigma_{m_{1}}^{2}, \sigma_{m_{2}}^{2}} \\ r_{\sigma_{m_{2}}^{2}, \sigma_{m_{1}}^{2}}^{2} & r_{\sigma_{m_{2}}, \sigma_{m_{2}}^{2}}^{2}\end{array}\right)$

where $r_{\sigma_{m_{i}}}^{2} \sigma_{m_{j}}^{2}$ represents second-order derivative of $r(\Gamma)$ with respect to $\sigma_{m_{i}}^{2}$ and $\sigma_{m_{j}}^{2}$. It can be shown that $r_{\sigma_{m_{1}}, \sigma_{m_{2}}^{2}}$ and $r_{\sigma_{m_{2}}, \sigma_{m_{1}}^{2}}$ are zero. Hence, the diagonal terms must be positive for the convexity of $r(\Gamma)$ with respect to $\sigma_{m_{1}}^{2}$ and $\sigma_{m_{2}}^{2}$. After some manipulation, $r_{\sigma_{m_{i}}^{2}, \sigma_{m_{i}}^{2}}$ can be expressed for $i \in\{1,2\}$ as

$r_{\sigma_{m_{i}}, \sigma_{m_{i}}^{2}}=\frac{\mu}{8 \sqrt{2 \pi}} \exp \left(-\frac{\mu^{2}}{8\left(\sigma_{x_{i}}^{2}+\sigma_{m_{i}}^{2}\right)}\right) \frac{1}{\left(\sigma_{x_{i}}^{2}+\sigma_{m_{i}}^{2}\right)^{5 / 2}}\left(\frac{\mu^{2}}{8\left(\sigma_{x_{i}}^{2}+\sigma_{m_{i}}^{2}\right)}-\frac{3}{2}\right)$.

From (27), the convexity condition for $r(\Gamma)$ can be obtained as $\frac{\mu^{2}}{\sigma_{x_{i}}^{2}+\sigma_{m_{i}}^{2}} \geq 12$ for $i=1,2$. That is, if this condition is satisfied for all values of $\sigma_{m_{i}}^{2}$ under the total cost constraint, the optimization problem becomes a convex optimization problem as the constraint is already convex as discussed previously.

Lemma 2 presents conditions under which the optimal cost allocation problem in (25) becomes a convex optimization problem. In that case, the problem can be solved based on convex optimization algorithms such as the interior-point algorithm [28].

\section{Cost allocation for Neyman-Pearson criterion}

The Bayesian criterion considered in the previous section is well-suited in the presence of prior probabilities of the hypotheses and cost assignments for possible decisions (see (1)-(3)). However, in some cases, the information about the prior probabilities of the hypotheses may not be available or assigning costs to possible decisions may not be suitable. In such scenarios, the NeymanPearson approach can be adopted for binary hypothesis-testing problems, where the aim is to maximize the probability of detection while satisfying a constraint on the probability of false alarm [25]. In this section, the Neyman-Pearson approach is employed for designing optimum centralized and decentralized detection systems in the presence of a cost constraint on measurement devices.

\subsection{Centralized detection}

As described in Section 2.1, the sensors in a centralized detection system transmit all of their observations to the fusion center and the fusion center decides on the hypothesis. Therefore, it suffices to apply the Neyman-Pearson criterion to the fusion center only. In this context, the aim is to maximize the probability of detection subject to the constraints on the probability of false alarm and the total cost, which is stated by the following optimization problem:

$\max _{\substack{\left.\sigma_{m_{i}^{2}}^{2}\right\}_{i=1}^{K} \\ \int_{\Gamma_{1}}}} p_{1}(\mathbf{y}) d \mathbf{y}$ subject to $\int_{\Gamma_{1}} p_{0}(\mathbf{y}) d \mathbf{y} \leq \alpha_{f c}, \quad \frac{1}{2} \sum_{i=1}^{K} \log _{2}\left(1+\frac{\sigma_{x_{i}}^{2}}{\sigma_{m_{i}}^{2}}\right) \leq C_{T}$,

where $\Gamma_{1}$ is the decision region for hypotheses $H_{1}, p_{i}(\mathbf{y})$ is the probability distribution of the observation under $H_{i}$, where $i \in\{0,1\}$, and $\alpha_{\mathrm{fc}}$ is the false alarm constraint. The solution of (28) yields the maximum value of the probability of detection via optimal cost assignments for the local sensors under the false alarm and total cost constraints.

Next, the Gaussian scenario is investigated as a special case based on the same distributions and assumptions employed in Section 2.1. Due to the presence of separate constraints in (28), the 
optimal NP decision rule can be obtained first, which leads to a likelihood ratio test with the probability of false alarm set to $\alpha_{\mathrm{fc}}$ [25]. For the considered Gaussian scenario, the corresponding probability of detection can be obtained as $P_{D}=Q\left(Q^{-1}\left(\alpha_{f c}\right)-d\right)$, where $d$ is given by (9) [25]. Therefore, the optimization problem in (28) can be expressed as follows:

$\max _{\left\{\sigma_{m_{i}}^{2}\right\}_{i=1}^{K}} Q\left(Q^{-1}\left(\alpha_{f c}\right)-d\right)$ subject to $\frac{1}{2} \sum_{i=1}^{K} \log _{2}\left(1+\frac{\sigma_{x_{i}}^{2}}{\sigma_{m_{i}}^{2}}\right) \leq C_{T}$

In order to maximize the objective function, the term inside $Q$ function should be minimized which can be achieved by increasing $d$ in (9). This results in the same optimization problem proposed in Section 2.1; hence, the cost values of the sensors are determined according to the algorithm given in (12).

\subsection{Decentralized detection}

In decentralized detection, all local sensors make their own decisions, which are processed in the fusion center to decide on the hypothesis. In Section 2.2, local sensors make a decision according to the Bayes rule and the majority fusion rule is employed at the fusion center. In this part, decisions are made according to the Neyman-Pearson criterion in the local sensors and the fusion center uses a counting rule [32]. The counting rule is specified in such a way that the probability of false alarm is lower than a specified threshold. As an example, the probability of false alarm in the fusion center versus the value of $N$ (for the $N$ out of $K$ rule) is illustrated in Fig. 3 for a sensor network with 12 local sensors. In the figure, the probability of false alarm for the local sensors is $10^{-3}$ and the measurements of the sensors are independent. For such a system to achieve an overall probability of false alarm lower than $10^{-12}$, the best fusion rule becomes 5 out of 12 . Moreover, it is observed that the probability of false alarm is a decreasing function of $N$ similar to the probability of detection. In order to achieve the maximum probability of detection, $N$ is chosen to be the minimum of possible value that satisfies constraint on the probability of false alarm, $\alpha_{\mathrm{fc}}$.

The same assumptions and the probability distributions used in Section 2.2 are employed in this section. Then, the probability of false alarm $P_{F A_{f C}}$ at the fusion center for the $N$ out of $K$ strategy is calculated as follows:

$P_{F A_{f c}}=\sum_{z=N}^{K} \sum_{c=1}^{\left(\begin{array}{c}K \\ z\end{array}\right)} \prod_{i=1}^{K}\left|l_{(z, c, i)}-1\right|+\left(2 l_{(z, c, i)}-1\right) \alpha_{i}$,

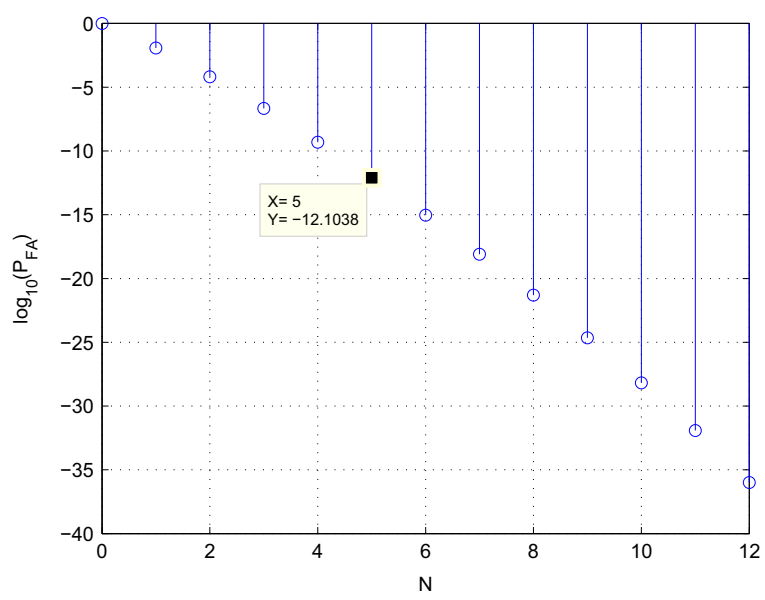

Fig. 3. Probability of false alarm versus $N$ for the $N$ out $K$ fusion rule. where $\alpha_{\mathrm{i}}$ is the probability of false alarm at the $i$ th sensor, and $l_{(z, c, i)}$ corresponds to the element at the $c$ th row and the $i$ th column of matrix $\mathbf{L}(z)$, as defined in Section 2.2.

The proposed optimization problem aims to maximize the probability of detection while keeping the total cost of the sensors under a certain limit and guaranteeing that the probability of false alarm is below the specified false alarm constraint. Based on (30), the optimization problem is stated as

$$
\begin{aligned}
& \max _{\left\{\sigma_{m_{i}}^{2}\right\}_{i=1}^{K}} \sum_{z=N}^{K} \sum_{c=1}^{\left(\begin{array}{c}
K \\
z
\end{array}\right)} \prod_{i=1}^{K}\left|l_{(z, c, i)}-1\right|+\left(2 l_{(z, c, i)}-1\right) P_{D_{i}} \\
& \text { subject to } \frac{1}{2} \sum_{i=1}^{K} \log _{2}\left(1+\frac{\sigma_{x_{i}}^{2}}{\sigma_{m_{i}}^{2}}\right) \leq C_{T},
\end{aligned}
$$

where $P_{D_{i}}$ is the probability of detection of the $i$ th sensor, and the value of $N$ is equal to the minimum integer number that satisfies $P_{F A_{f c}} \leq \alpha_{f c}$ for the $N$ out of $K$ decision rule.

As a special case, the Gaussian scenario in Section 2.2 is investigated. In this case, the detection threshold is calculated based on the given $\alpha_{\mathrm{i}}$ value by equating the probability of false alarm to $\alpha_{\mathrm{i}}$. Then, the probability of detection is determined for the obtained detection threshold. In particular, the probability of detection for the $i$ th sensor is calculated as follows:

$P_{D_{i}}=Q\left(Q^{-1}\left(\alpha_{i}\right)-\frac{\mu_{i 1}-\mu_{i 0}}{\sqrt{\sigma_{x_{i}}^{2}+\sigma_{m_{i}}^{2}}}\right)$.

From (32), the optimization problem in (31) can be specified as follows:

$$
\begin{aligned}
& \max _{\left\{\sigma_{m_{i}}^{2} i_{i=1}^{K}\right.} \sum_{z=N}^{K} \sum_{c=1}^{\left(\begin{array}{c}
K \\
z
\end{array}\right)} \prod_{i=1}^{K} \|_{(z, c, i)}-1 \mid+\left(2 l_{(z, c, i)}-1\right) Q\left(Q^{-1}\left(\alpha_{i}\right)-\frac{\mu_{i 1}-\mu_{i 0}}{\sqrt{\sigma_{x_{i}}^{2}+\sigma_{m_{i}}^{2}}}\right) \\
& \text { subject to } \frac{1}{2} \sum_{i=1}^{K} \log _{2}\left(1+\frac{\sigma_{x_{i}}^{2}}{\sigma_{m_{i}}^{2}}\right) \leq C_{T},
\end{aligned}
$$

where $N$ is chosen as stated above. Exhaustive search with parallel computing is used to solve the optimization problem as in ((23). The solution of (33) results in the maximum probability of detection for the given cost and false alarm constraints.

Remark 2. Neyman-Pearson hypothesis testing is employed at the local sensors and the optimization problem is formulated for the given fusion rule, which is the counting rule. As another approach, the optimization problem can be formulated over the fusion rule, local thresholds and measurement noise variances. Although the latter optimization problem can lead to improved performance, its computational complexity is significantly higher than that of the former one.

\section{Cost allocation for J-divergence criterion}

As alternatives to the Bayesian and NP criteria, distance related bounds can be used for quantifying detection performance. The distance related bounds provide upper and lower bounds on the probabilities of detection and false alarm (or, the probability of error). Some examples of these bounds are the Bhattacharrya bound, J-divergence and Chernoff bound [25]. These bounds belong to the Ali-Silvey class of distance measures [33]. In this section, we employ J-divergence, firstly introduced by Jeffreys [34], for the cost allocation problem. The $J$-divergence is a commonly used metric for detection performance [35-38]. It introduces a lower bound on the probability of error $P_{e}$ [37] as follows: 
$P_{e}>\pi_{0} \pi_{1} e^{-J / 2}$,

where $\pi_{0}$ and $\pi_{1}$ are the prior probabilities of hypothesis $H_{0}$ and hypothesis $H_{1}$, respectively, and $J$ denotes the $J$-divergence, which is the symmetric version of the Kullback-Leibler (KL) distance [39]. The $J$-divergence is defined between two probability densities, $p$ and $q$, as follows:

$J(p, q)=D(p \| q)+D(q \| p)$,

where $D(p \| q)$ is the KL distance between $p$ and $q$, which is calculated as

$D(p \| q)=\int p(x) \ln \frac{p(x)}{q(x)} d x$.

According to the formula in (36), the $J$-divergence is obtained as follows:

$J(p, q)=\int(p(x)-q(x)) \ln \frac{p(x)}{q(x)} d x$.

In this section, the cost allocation problem is investigated based on the $J$-divergence criterion for both centralized and decentralized detection systems.

\subsection{Centralized detection}

The aim is to maximize the detection performance at the fusion center under a total cost constraint. To this aim, the $J$-divergence between $p_{1}(\mathbf{y})$ and $p_{0}(\mathbf{y})$ is to be maximized. The optimization problem for centralized detection can be written as follows:

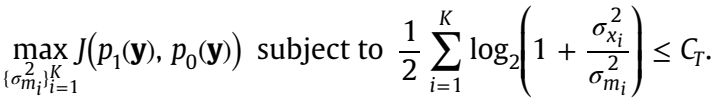

Although the $J$-divergence is useful especially in cases where the error probabilities cannot easily be evaluated, it is a metric that can be employed in any scenario. Since the Gaussian distribution is commonly encountered in practice, (38) is investigated for the Gaussian scenario in detail as in the previous section. (The $J$-divergence for two Gaussian distributions is considered for detection performance optimization problems in the literature; e.g., [35].) The $J$-divergence between densities $p$ and $q$ with distributions $\mathcal{N}\left(\boldsymbol{\mu}_{\mathbf{0}}, \boldsymbol{\Sigma}_{\mathbf{0}}\right)$ and $\mathcal{N}\left(\boldsymbol{\mu}_{\mathbf{1}}, \boldsymbol{\Sigma}_{\mathbf{1}}\right)$, respectively, is given as follows [40]:

$$
\begin{aligned}
J(p, q)= & \frac{1}{2}\left(\boldsymbol{\mu}_{\mathbf{1}}-\boldsymbol{\mu}_{\mathbf{0}}\right)^{T}\left(\boldsymbol{\Sigma}_{\mathbf{0}}^{-1}+\boldsymbol{\Sigma}_{\mathbf{1}}^{-1}\right)\left(\boldsymbol{\mu}_{\mathbf{1}}-\boldsymbol{\mu}_{\mathbf{0}}\right) \\
& +\frac{1}{2} \operatorname{tr}\left\{\boldsymbol{\Sigma}_{\mathbf{0}}^{-1} \boldsymbol{\Sigma}_{\mathbf{1}}+\boldsymbol{\Sigma}_{\mathbf{1}}^{-1} \boldsymbol{\Sigma}_{\mathbf{0}}-2 \mathbf{I}\right\},
\end{aligned}
$$

where $\mathbf{I}$ is the identity matrix with the same size as the covariance matrices. For the Gaussian scenario described in Section 2.1, the $J$ divergence is calculated as

$J\left(p_{1}(\mathbf{y}), p_{0}(\mathbf{y})\right)=\left(\boldsymbol{\mu}_{\mathbf{1}}-\boldsymbol{\mu}_{\mathbf{0}}\right)^{T} \boldsymbol{\Sigma}_{\mathbf{T}}^{-1}\left(\boldsymbol{\mu}_{\mathbf{1}}-\boldsymbol{\mu}_{\mathbf{0}}\right)$,

which is the same as the objective function in (11). Therefore, the same optimization problem as in Sections 2.1 and 3.1 is obtained. As a result, the cost allocation strategy is determined according to the algorithm in (12).

\subsection{Decentralized detection}

In this part, a decentralized detection system is examined based on the $J$-divergence criterion. The aim is to maximize the $J$ divergence between $p_{1}(\mathbf{u})$ and $p_{0}(\mathbf{u})$ under a total cost constraint. The mathematical description of the problem is given by $\max _{\left\{\sigma_{m_{i}}^{2}\right\}_{i=1}^{K}} J\left(p_{1}(\mathbf{u}), p_{0}(\mathbf{u})\right)$ subject to $\frac{1}{2} \sum_{i=1}^{K} \log _{2}\left(1+\frac{\sigma_{x_{i}}^{2}}{\sigma_{m_{i}}^{2}}\right) \leq C_{T}$.

In order to solve this problem, the conditional density functions of the local decisions should be determined. These densities are given as follows:

$p_{1}(\mathbf{u})=\prod_{i=1}^{K} P_{D_{i}}^{u_{i}}\left(1-P_{D_{i}}\right)^{1-u_{i}}$

$p_{0}(\mathbf{u})=\prod_{i=1}^{K} P_{F A_{i}}^{u_{i}}\left(1-P_{F A_{i}}\right)^{1-u_{i}}$,

where $P_{F A_{i}}$ and $P_{D_{i}}$ represent the probability of false alarm and the probability of detection at the $i$ th sensor, respectively. The information about $P_{F A_{i}}$ and $P_{D_{i}}$ can be obtained by using the NeymanPearson rule. The objective function in the optimization problem can be expressed as follows:

$$
\begin{aligned}
J\left(p_{1}(\mathbf{u}), p_{0}(\mathbf{u})\right)= & \sum_{u_{1}=0}^{1} \sum_{u_{2}=0}^{1} \ldots \sum_{u_{k}=0}^{1}\left(\prod_{i=1}^{K} P_{D_{i}}^{u_{i}}\left(1-P_{D_{i}}\right)^{1-u_{i}}\right. \\
& \left.-\prod_{i=1}^{K} P_{F A_{i}}^{u_{i}}\left(1-P_{F A_{i}}\right)^{1-u_{i}}\right) \ln \frac{\prod_{i=1}^{K} P_{D_{i}}^{u_{i}}\left(1-P_{D_{i}}\right)^{1-u_{i}}}{\prod_{i=1}^{K} P_{F A_{i}}^{u_{i}}\left(1-P_{F A_{i}}\right)^{1-u_{i}}} .
\end{aligned}
$$

In order to examine the Gaussian scenario, $P_{D_{i}}$ is determined in terms of the specified probability of false alarm as in (32). Then, the given $P_{F A_{i}}$ and the calculated $P_{D_{i}}$ values can be inserted into (44) in order to determine the $J$-divergence between $p_{1}(\mathbf{u})$ and $p_{0}(\mathbf{u})$. At this point, the obtained $J$-divergence between $p_{1}(\mathbf{u})$ and $p_{0}(\mathbf{u})$ is inserted into (41) and the optimization problem is solved numerically in order to obtain the optimum detection performance in the sense of $J$-divergence. As the numerical solution approach in the next section, exhaustive search is employed.

\section{Numerical results}

In this section, the performance of the proposed optimal cost allocation strategies is evaluated via numerical examples. Firstly, the results for centralized detection in the Bayesian framework are presented. The distribution of the observation $\mathbf{x}$ under hypothesis $H_{0}$ is given by $\mathcal{N}(\mathbf{0}, \mathbf{\Sigma})$, where $\mathbf{0}=[0,0,0]^{T}$. Similarly, the distribution of $\mathbf{x}$ under hypothesis $H_{1}$ is modeled as $\mathcal{N}(\mathbf{1}, \boldsymbol{\Sigma})$, where $\mathbf{1}=[1,1,1]^{T}$. In these distributions, $\boldsymbol{\Sigma}$ represents the covariance matrix, which is expressed as $\operatorname{diag}\left\{\sigma_{x_{1}}^{2}, \sigma_{x_{2}}^{2}, \sigma_{x_{3}}^{2}\right\}$. The values of the variances $\sigma_{x_{1}}^{2}, \sigma_{x_{2}}^{2}$ and $\sigma_{x_{3}}^{2}$ are set to $0.2,0.7$, and 1.2 , respectively. Measurement noise $\mathbf{m}$ also has Gaussian distribution denoted by $\mathcal{N}\left(\mathbf{0}, \boldsymbol{\Sigma}_{\mathbf{m}}\right)$, where $\boldsymbol{\Sigma}_{\mathbf{m}}=\operatorname{diag}\left\{\sigma_{m_{1}}^{2}, \sigma_{m_{2}}^{2}, \sigma_{m_{3}}^{2}\right\}$. Lastly, the hypotheses are equally likely; i.e., $\pi_{0}=\pi_{1}=0.5$.

The strategies that are compared with the proposed optimal cost allocation strategy are

- assignment of equal measurement variances to the measurement devices (sensors), and

- assignment of all the cost to the sensor with the best observation.

When the measurement devices have equal measurement noise variances; i.e., $\sigma_{m}^{2}=\sigma_{m_{1}}^{2}=\sigma_{m_{2}}^{2}=\sigma_{m_{3}}^{2}$, the variance $\sigma_{m}{ }^{2}$ can be calculated by using the formula $\prod_{i=1}^{3}\left(1+\sigma_{x_{i}}^{2} / \sigma_{m}^{2}\right)=2^{2 C_{T}}$, where the variance $\sigma_{m}{ }^{2}$ corresponds to the smallest positive root of this 


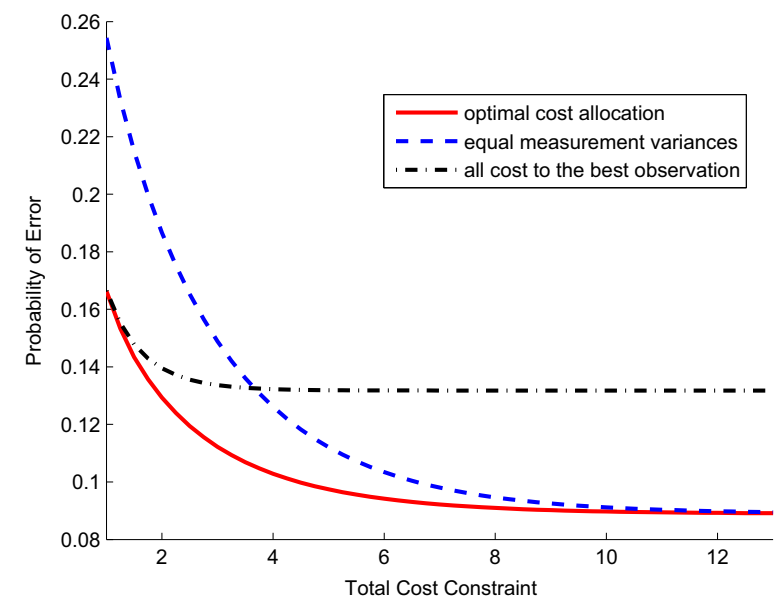

Fig. 4. Probability of error vs. total cost constraint for Bayesian centralized detection.

equation. After finding $\sigma_{m}{ }^{2}$, the probability of error is calculated as $r\left(\delta_{B}\right)=Q\left(0.5 \sqrt{\sum_{i=1}^{3} 1 /\left(\sigma_{x_{i}}^{2}+\sigma_{m}^{2}\right)}\right)$. In the second strategy, all the available cost is assigned to the measurement device having the observation with the smallest variance. In this example, $\sigma_{x_{1}}^{2}$ has the smallest variance; hence, all the cost is assigned to sensor 1 and $\sigma_{m_{1}}^{2}=\sigma_{x_{1}}^{2} /\left(2^{2 C_{T}}-1\right)$. The other variances $\sigma_{m_{2}}^{2}$ and $\sigma_{m_{3}}^{2}$ are set to infinity, and no measurements are taken from the corresponding measurement devices. The probability of error is obtained for this case as $r\left(\delta_{B}\right)=Q\left(0.5 \sqrt{2^{2 G_{T}}-1} / \sqrt{2^{2 C_{T}} \sigma_{x_{1}}^{2}}\right)$. The results obtained for the centralized detection in the Bayesian framework are presented in Fig. 4, which illustrates the probability of error versus the total cost constraint, $C_{T}$, for the optimal cost allocation strategy and the two strategies described above. For small values of $C_{T}$, assigning all the cost to the sensor with the best observation converges the optimal solution since, when $C_{T}$ is small, the optimal strategy allocates the total cost to the sensors with the best observations. Moreover, the probability of error for assigning all the cost to the sensor with the best observation converges to $Q\left(0.5 / \sqrt{\sigma_{x_{1}}^{2}}\right)$, which is equal to $Q(0.5 / \sqrt{0.2})=0.1318$ since $\sigma_{m_{1}}^{2}$ goes to zero as $C_{T}$ increases. For high total cost constraints, the equal measurement variances strategy converges to the optimal strategy. Similar to the strategy that assigns all the cost to the sensor with the best observation, when $C_{T}$ is high, the measurement noise variances become low and the probability of error converges to $r\left(\delta_{B}\right)=Q\left(0.5 \sqrt{1 / \sigma_{x_{1}}^{2}+1 / \sigma_{x_{2}}^{2}+1 / \sigma_{x_{3}}^{2}}\right)$ which is equal to 0.0889 for the values specified above. Overall, the proposed optimal cost allocation strategy yields the lowest probabilities of error. In other words, the optimum performance according to the Bayesian criterion is attained with the optimal cost allocation strategy.

For the same setting as in Fig. 4, the results for decentralized detection in the Bayesian framework are presented in Fig. 5. As observed from Fig. 5, assigning all the cost to the sensor with the best observation yields the worst performance in this case since all the sensors make their own decisions. When zero cost is assigned to a sensor, the measurement noise variance becomes infinity and the probability of error for that measurement device becomes 0.5 . Then, the probability of error converges to $r(\Gamma)=0.75 Q\left(0.5 / \sqrt{\sigma_{x_{1}}^{2}}\right)$ $+0.5 Q\left(-0.5 / \sqrt{\sigma_{x_{1}}^{2}}\right)$ for high cost constraints. For $\sigma_{x_{1}}^{2}=0.2$, the probability of error converges to 0.3159 . When the cost constraint is high, the equal measurement variances strategy converges to the optimal strategy. For high cost constraints, the probability of error for the equal measurement variances strategy converges to

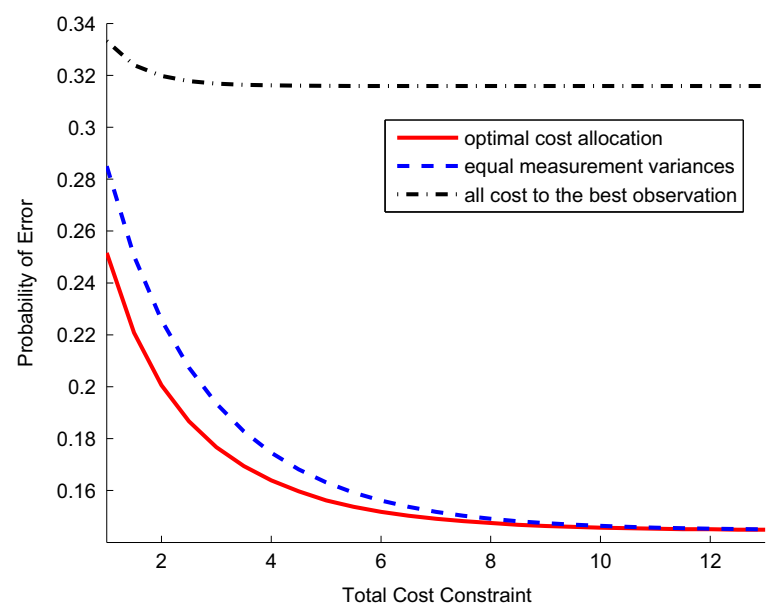

Fig. 5. Probability of error vs. total cost constraint for Bayesian decentralized detection.

$r(\Gamma)=a b+a c+b c-2 a b c$ where $a=Q\left(0.5 / \sqrt{\sigma_{x_{1}}^{2}}\right), b=Q\left(0.5 / \sqrt{\sigma_{x_{2}}^{2}}\right)$, and $c=Q\left(0.5 / \sqrt{\sigma_{x_{3}}^{2}}\right)$. For the values specified above, $r(\Gamma)$ converges to 0.1446 . Overall, the optimal cost allocation strategy yields the lowest probabilities of error for decentralized detection, as well.

In the Neyman-Pearson framework, the probability of detection achieved by the proposed algorithm is compared with the two strategies explained above (that is, assignment of equal measurement variances to the measurement devices and assignment of all the cost to the sensor with the best observation). In centralized detection, the distribution of observation $\mathbf{x}$ is specified by $\mathcal{N}(\mathbf{0}, \boldsymbol{\Sigma})$ and $\mathcal{N}(\mathbf{2}, \mathbf{\Sigma})$ for hypotheses $H_{0}$ and $H_{1}$, respectively. The covariance matrix is the same as in the previous scenario; i.e., $\boldsymbol{\Sigma}=\operatorname{diag}\{0.2,0.7,1.2\}$. The probability of false alarm at the fusion center is required to be less than or equal to $\alpha_{f c}=10^{-6}$. The results obtained for centralized detection in the Neyman-Pearson framework are presented in Fig. 6. Similar to the results for the Bayesian criterion, assigning all the cost to the best observation yields similar performance to the optimal algorithm for low cost values. When the cost budget increases, $P_{D}$ converges to $Q\left(Q^{-1}\left(\alpha_{f c}\right)-\mu_{1} / \sigma_{x_{1}}\right)$; hence, for the considered parameters, the probability of detection converges to $Q\left(Q^{-1}\left(10^{-6}\right)-2 / \sqrt{0.2}\right)=0.3892$. On the other hand, the equal measurement variances strategy converges to the same value of $Q\left(Q^{-1}\left(\alpha_{f c}\right)-\sqrt{\mu_{1}^{2} / \sigma_{x_{1}}^{2}+\mu_{2}^{2} / \sigma_{x_{2}}^{2}+\mu_{3}^{2} / \sigma_{x_{3}}^{2}}\right)$ as the optimal algorithm for high cost values. In particular, the optimal algorithm converges to $Q\left(Q^{-1}\left(10^{-6}\right)-\sqrt{4 / 0.2+4 / 0.7+4 / 1.2}\right)=0.7377$ as the total cost

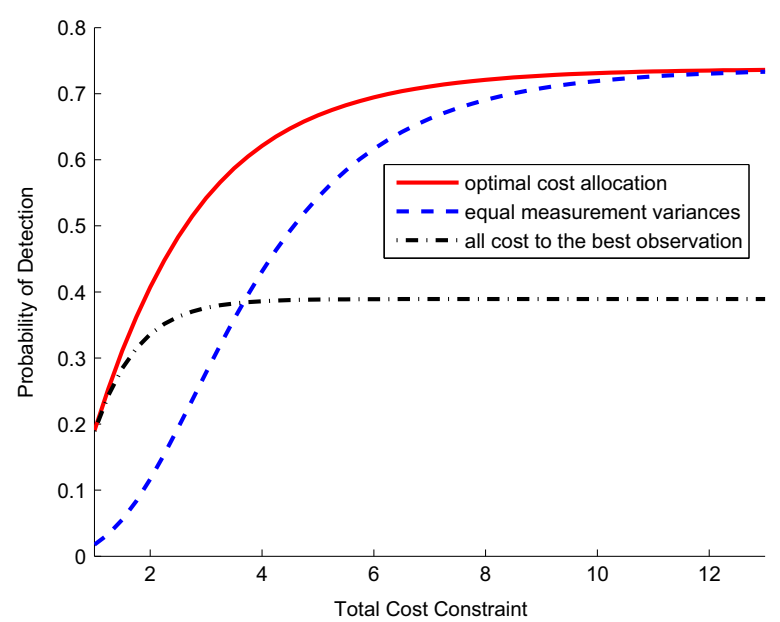

Fig. 6. Probability of detection vs. total cost constraint for NP centralized detection. 


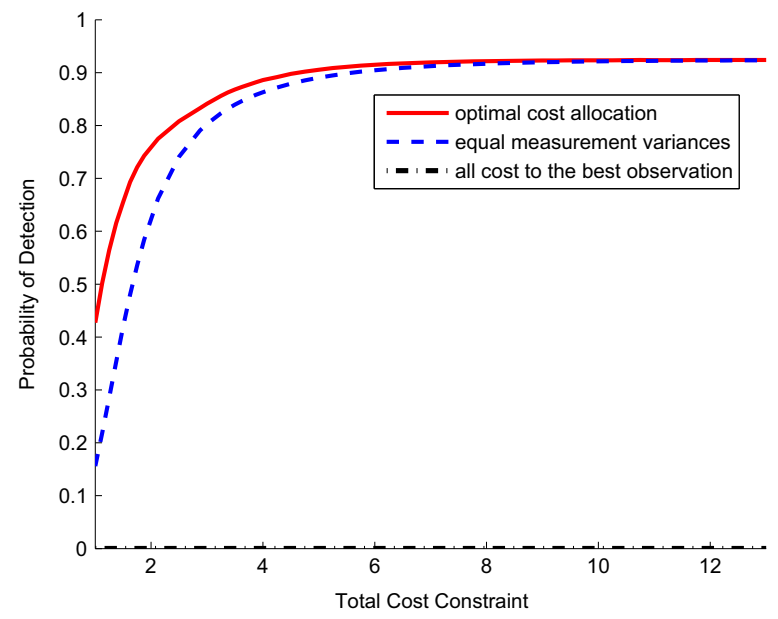

Fig. 7. Probability of detection vs. total cost constraint for NP decentralized detection.

constraint increases. As a result, the optimal cost allocation strategy produces the maximum probability of detection in all cases and outperforms the other approaches.

In the next example, the optimality of the proposed algorithm is illustrated for decentralized detection in the Neyman-Pearson framework. The distribution of observation $\mathbf{x}$ is denoted as $\mathcal{N}(\mathbf{0}, \boldsymbol{\Sigma})$ and $\mathcal{N}(\mathbf{4}, \boldsymbol{\Sigma})$ for hypotheses $H_{0}$ and $H_{1}$, respectively, where $\boldsymbol{\Sigma}$ is the same as that in the centralized detection case. All the local sensors have the same probability of false alarm given by $\alpha_{1}=\alpha_{2}=\alpha_{3}=10^{-4}$. It is required to achieve a false alarm probability not exceeding $10^{-7}$ at the fusion center. In order to satisfy this false alarm probability at the fusion center, the 2 out 3 fusion rule must be used. This fusion rule produces a false alarm probability of $10^{-7.5}$, which satisfies the requirement. The results related to this scenario are shown in Fig. 7. It is observed that assigning all the cost to the best observation has detection probability close to zero since the sensors having zero cost have infinite noise powers and the probability of detection for these sensors is $10^{-4}$. When the total cost constraint is high, the equal measurement variances strategy and the proposed algorithm converge to the same probability of detection, specified by $P_{D}=P_{d_{1}} P_{d_{2}}+P_{d_{1}} P_{d_{3}}+P_{d_{2}} P_{d_{3}}-2 P_{d_{1}} P_{d_{2}} P_{d_{3}}$, where $P_{d_{1}}=Q\left(Q^{-1}\left(\alpha_{1}\right)-\mu_{1} / \sigma_{x_{1}}\right)$, $P_{d_{2}}=Q\left(Q^{-1}\left(\alpha_{2}\right)-\mu_{2} / \sigma_{x_{2}}\right)$ and $P_{d_{3}}=Q\left(Q^{-1}\left(\alpha_{3}\right)-\mu_{3} / \sigma_{x_{3}}\right)$. For the values given above, $P_{D}$ converges to 0.9240 . Overall, the optimal cost allocation algorithm yields the highest probabilities of detection in this scenario.

Next, the $J$-divergence criterion is considered and the proposed algorithm is compared with the other two strategies. In centralized detection, the distribution of observation vector $\mathbf{x}$ is represented by $\mathcal{N}(\mathbf{0}, \boldsymbol{\Sigma})$ and $\mathcal{N}(\mathbf{2}, \boldsymbol{\Sigma})$ for hypotheses $H_{0}$ and $H_{1}$, respectively, where the covariance matrix is given by $\boldsymbol{\Sigma}=\operatorname{diag}\{0.2,0.7,1.2\}$. The results for this case are shown in Fig. 8. It is observed that assigning all the cost to the best observation and the proposed optimal strategy achieve similar performance for low cost values. When the total cost increases, the $J$-divergence converges to $\mu_{1}^{2} / \sigma_{x_{1}}^{2}=20$ for the strategy that assigns all the cost to the best observation, which is significantly lower than that achieved by the optimal strategy. On the other hand, the performance of the equal measurement variances strategy converges to that of the optimal algorithm for high cost values; in particular, the $J$-divergence converges to $\sum_{i=1}^{3} \mu_{i}^{2} / \sigma_{x_{i}}^{2}=29.0476$. Overall, the proposed algorithm yields the maximum $J$-divergence for all cost values resulting in the optimum performance.

In the final example, a decentralized detection problem is considered according to the $J$-divergence criterion. The distribution of observation $\mathbf{x}$ is denoted by $\mathcal{N}(\mathbf{0}, \boldsymbol{\Sigma})$ and $\mathcal{N}(\mathbf{4}, \boldsymbol{\Sigma})$ for hypotheses $H_{0}$

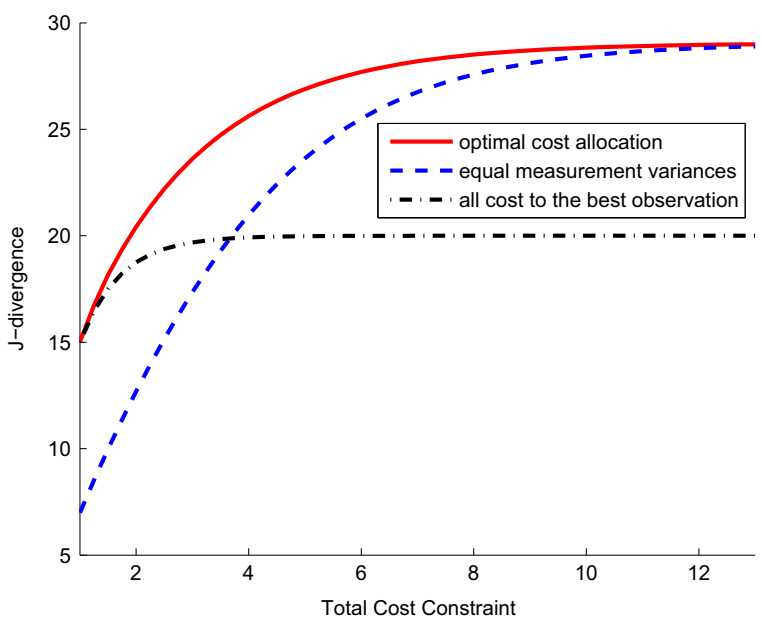

Fig. 8. J-divergence versus the total cost constraint for centralized detection.

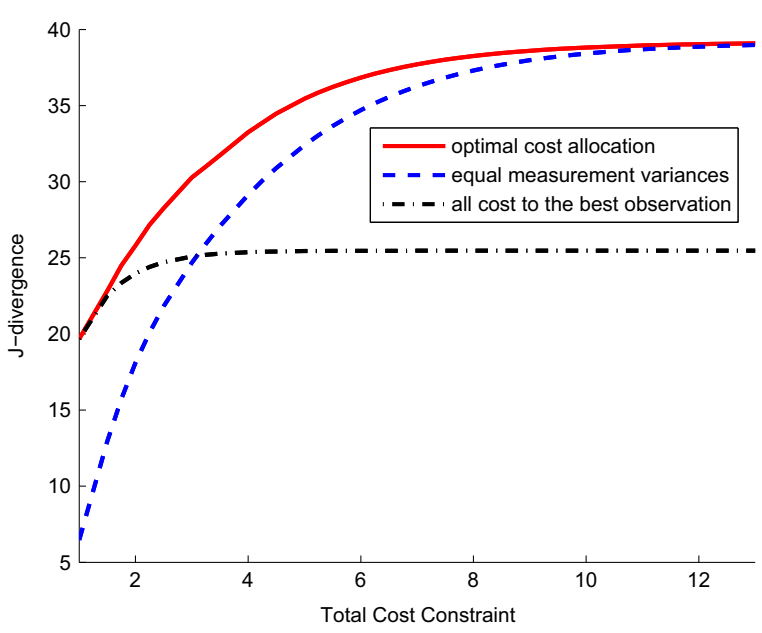

Fig. 9. J-divergence vs. total cost constraint for decentralized detection.

and $H_{1}$, respectively, where $\boldsymbol{\Sigma}$ is the same as in the centralized detection case. The probability of false alarm for the local sensors is given by $\alpha_{1}=\alpha_{2}=\alpha_{3}=10^{-4}$. The results related to this scenario are presented in Fig. 9. It is noted that assigning all the cost to the best observation achieves improved performance in this case compared to the decentralized detection examples in the Bayesian and Neyman-Pearson frameworks Figs. 5 and 7, respectively. The main reason for this observation is that no counting rule is applied at the fusion center in this case. Similar to the centralized detection case, the proposed algorithm and the algorithm that assigns all the cost to the best observation yield similar results for low cost values. As the cost increases, the equal measurement variance strategy and the proposed algorithm converges to the same value of 39.177 while assigning all the cost to the best observation leads to a convergence to 25.466 for high cost values. From Fig. 9, it is observed that the proposed algorithm yields the maximum $J$-divergence in all the cases, and achieves the optimum detection performance.

\section{Conclusions}

In this manuscript, centralized and decentralized detection systems have been investigated in the presence of cost constrained measurements. Novel cost allocation strategies that achieve the optimum detection performance according to the Bayesian, Neyman-Pearson and $J$-divergence criteria have been proposed for both centralized and 
decentralized detection systems. A closed form expression has been presented for the measurement noise variances by considering centralized detection in a Gaussian scenario. This expression indicates that if the observation variance is low, using a measurement device with a high cost is more beneficial. Also, the convexity property of the objective and constraint functions has been studied under certain conditions. For decentralized detection, a general probability of error expression for the Bayesian criterion and the probabilities of detection and false alarm expressions for the Neyman-Pearson framework have been presented according to the counting rules at the fusion center. In addition, the $J$-divergence has been employed for the distance based criterion. The Gaussian scenario has been investigated as a special case and the optimization problems have been proposed for all the criteria. The optimality of the proposed cost allocation strategies has been shown via numerical examples. Overall, the proposed cost allocation strategies minimize the Bayes risk for the Bayesian criterion, maximize the probability of detection (under a constraint on the probability of false alarm) for the Neyman-Pearson criterion, and maximize the $J$ divergence for the distance based criterion under given cost constraints, and they achieve the optimum performance.

\section{References}

[1] C. Xu and S. Kay, On centralized composite detection with distributed sensors, in IEEE Radar Conference, May 2008.

[2] J.N. Tsitsiklis, Decentralized detection, Adv. Stat. Signal Process. 2 (1993) $297-344$.

[3] A. Ozcelikkale, H.M. Ozaktas, E. Arikan, Signal recovery with cost-constrained measurements, IEEE Trans. Signal Process. 58 (July) (2010) 3607-3617.

[4] B. Dulek, S. Gezici, Cost minimization of measurement devices under estimation accuracy constraints in the presence of Gaussian noise, Digit. Signal Process. 22 (2012) 828-840.

[5] K. Liu and A.M. Sayeed, Optimal distributed detection strategies for wireless sensor networks, in Proceedings of the 42nd Annual Allerton Conference on Communications, Control and Computing, October 2004.

[6] J.F. Chamberland, V. Veeravalli, Decentralized detection in sensor networks, IEEE Trans. Signal Process. 51 (February) (2003) 407-416.

[7] W.P. Tay, Decentralized detection in resource-limited sensor network architectures, Ph.D. Thesis, Massachusetts Institute of Technology, 2008.

[8] J.F. Chamberland, V.V. Veeravalli, Asymptotic results for decentralized detection in power constrained wireless sensor networks, IEEE J. Select. Areas Commun. 22 (August) (2004) 1007-1015.

[9] S. Appadwedula, V.V. Veeravalli, D.L. Jones, Energy-efficient detection in sensor networks, IEEE J. Select. Areas Commun. 23 (April) (2005) 693-702.

[10] C. Rago, P. Willett, Y. Bar-Shalom, Censoring sensors: a low-communicationrate scheme for distributed detection, IEEE Trans. Aerosp. Electron. Syst. 32 (April) (1996) 554-568.

[11] M. Lazaro, M. Sanchez-Fernandez, A. Artes-Rodriguez, Optimal sensor selection in binary heterogeneous sensor networks, IEEE Trans. Signal Process. 57 (April) (2009) 1577-1587.

[12] X. Zhang, H.V. Poor, M. Chiang, Optimal power allocation for distributed detection over mimo channels in wireless sensor networks, IEEE Trans. Signal Process. 56 (September) (2008) 4124-4140.

[13] R. Ahlswede, I. Csiszar, Hypothesis testing with communication constraints, IEEE Trans. Inf. Theory 32 (July) (1986) 533-542.

[14] S.K. Jayaweera, Large system decentralized detection performance under communication constraints, IEEE Commun. Lett. 9 (September) (2005) $769-771$

[15] S.K. Jayaweera, Bayesian fusion performance and system optimization for distributed stochastic Gaussian signal detection under communication constraints, IEEE Trans. Signal Process. 55 (April) (2007) 1238-1250.

16] S.K. Jayaweera, Decentralized detection of stochastic signals in power-constrained sensor networks, in: IEEE 6th Workshop on Signal Processing Advances in Wireless Communications, June 2005, pp. 270-274.

[17] S.K. Jayaweera, Sensor system optimization for Bayesian fusion of distributed stochastic signals under resource constraints, in: IEEE International Conference on Acoustics, Speech and Signal Processing (ICASSP), vol. 4, May 2006, pp. IV149-IV152.

[18] K.A.A. Tarzai, S.K. Jayaweera, and V. Aravinthan, Performance of decentralized detection in a resource-constrained sensor network with non-orthogonal communications, in: Conference Record of the Thirty-Ninth Asilomar Conference on Signals, Systems and Computers, pp. 437-441, October 2005.

[19] S.A. Aldosari and J.M.F. Moura, Fusion in sensor networks with communication constraints, in: Third International Symposium on Information Processing in Sensor Networks (IPSN), April 2004, pp. 108-115.

[20] B. Dulek, S. Gezici, Average fisher information maximisation in presence of cost-constrained measurements, Electron. Lett. 47 (May) (2011) 654-656.

21] S.P. Chepuri, G. Leus, Sparse sensing for distributed detection, IEEE Trans. Signal Process. 64 (March) (2016) 1446-1460.

[22] D. Bajovic, B. Sinopoli, J. Xavier, Sensor selection for event detection in wireless sensor networks, IEEE Trans. Signal Process. 59 (October) (2011) 4938-4953.

[23] C. Bruni, G. Koch, F. Papa, Estimate accuracy versus measurement cost saving in continuous time linear filtering problems, J. Frankl. Inst. 350 (5) (2013) 1051-1074.

[24] M.E. Tutay, S. Gezici, H. Soganci, O. Arikan, Optimal channel switching ove Gaussian channels under average power and cost constraints, IEEE Trans. Commun. 63 (May) (2015) 1907-1922.

[25] H.V. Poor, An Introduction to Signal Detection and Estimation, Springer-Verlag, New York, 1994.

[26] R.V. Hartley, Transmission of information, Bell Syst. Techn. J. 7 (3) (1928) 535-563.

[27] C.E. Shannon, A mathematical theory of communication, Bell Syst. Techn. J. 27 (October) (1948), 379-423,623-656.

[28] S. Boyd, L. Vandenberghe, Convex Optimization, Cambridge University Press, Cambridge, UK, 2004.

[29] R.T. Rockafellar, Convex Analysis, Princeton University Press; Princeton, New Jersey, USA, 1970.

[30] G. Ferrari and R. Pagliari, Decentralized detection in sensor networks with noisy communication links, in: Distributed Cooperative Laboratories: Networking, Instrumentation, and Measurements, Springer, New York, 2006, pp. 233-249.

[31] Q. Zhang, P. Varshney, and R. Wesel, Optimal distributed binary hypothesis testing with independent identical sensors, in: Conference of the Information Sciences and Systems, March 2000.

[32] B. Ahsant, R. Viswanathan, S. Jeyaratnam, and S. Jayaweera, New results on large sample performance of counting rules, in: 50th Annual Allerton Conference on Communication, Control, and Computing, October 2012, pp. 882885.

[33] S.M. Ali, S.D. Silvey, A general class of coefficients of divergence of one distribution from another, J. R. Stat. Soc. Ser. B (Methodol.) (1966) 131-142.

[34] H. Jeffreys, An invariant form for the prior probability in estimation problems, in: Proc. R. Soc. Lond. A Math. Phys. Eng. Sci. 186 (1946) 453-461.

[35] T. Kailath, The divergence and Bhattacharyya distance measures in signal selection, IEEE Trans. Commun. Technol. 15 (February) (1967) 52-60.

[36] H. Kobayashi, Distance measures and asymptotic relative efficiency, IEEE Trans. Inf. Theory 16 (May) (1970) 288-291.

[37] H. Kobayashi and J.B. Thomas, Distance measures and related criteria, in: Proceedings of the 5th Annual Allerton Conference on Circuit System Theory, October 1967, pp. 491-500.

[38] H. Poor, J. Thomas, Applications of Ali-Silvey distance measures in the design generalized quantizers for binary decision systems, IEEE Trans. Commun. 25 (September) (1977) 893-900.

[39] T.M. Cover, J.A. Thomas, Elements of Information Theory, Wiley, New York 1991.

[40] K.T. Abou-Moustafa and F.P. Ferrie, Modified divergences for Gaussian densities, in Structural, Syntactic, and Statistical Pattern Recognition, Springer, 2012, pp. 426-436. 